$>$ La croissance des os longs se fait selon un processus complexe impliquant la migration et la condensation de cellules mésenchymateuses en cellules chondrogéniques qui se différencient en chondrocytes produisant la matrice cartilagineuse pour former la plaque de croissance. De nombreux facteurs protéiques sont impliqués dans la régulation de ces phénomènes parmi lesquels des facteurs transcriptionnels, des facteurs de signalisation et des protéines de la matrice extracellulaire dont le rôle a été révélé grâce aux études de génétique moléculaire sur des dysplasies osseuses humaines et à la création de modèles animaux reproduisant certaines de ces maladies. Cet article se focalise sur deux récepteurs, FGFR3 et PTHRI, dont l'importance dans la croissance des os longs est illustrée par le groupe de dysplasies osseuses qui leurs sont associées. Des résultats récents indiquent que prolifération et différenciation chondrocytaires sont étroitement liées et que la croissance harmonieuse des os longs repose sur un équilibre strict entre différentes voies de signalisation dont celles contrôlées par ces facteurs. <

\section{Dysplasies \\ osseuses \\ héréditaires \\ et voies de \\ signalisation \\ associées aux \\ récepteurs FGFR3 \\ et PTHR 1}

Jacky Bonaventure, Caroline Silve

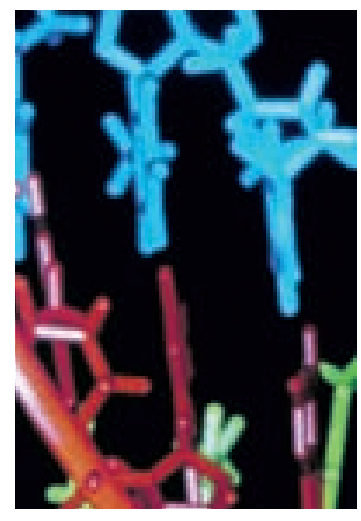

dans plusieurs gènes
J. Bonaventure: CNRS UMR 146, Institut Curie, Bâtiment 110, Centre Universitaire Paris Sud, 91405 Orsay, France.

C. Silve : Inserm U.426, Faculté de Médecine Xavier Bichat, 16, rue Henri Huchard, 75870 Paris, France.

caroline.silve@bichat.inserm.fr (exemple: dysplasie polyépiphysaire) (Tableau I) [2]. Enfin, certaines de ces pathologies sont associées à des anomalies du métabolisme des ions calcium et phosphate essentiels au développement et au maintien de la structure du cartilage et de l'os. La multiplicité des gènes et la complexité des systèmes de régulation mis en jeu dans les dysplasies osseuses sont telles qu'il serait illusoire de prétendre en faire une synthèse exhaustive dans un seul article. Nous avons donc choisi de présenter les mécanismes généraux régulant le développement du squelette, de décrire les étapes et les principaux facteurs contrôlant le développement des membres et de nous focaliser sur les chondrodysplasies associées à des anomalies des voies de signalisation régies par le FGFR3 (fibroblast growth factor receptor-3) et le PTHRI (récepteur de I'hormone parathyroïdienne, PTH et de son peptide apparenté, PTHrP, encore appelé récepteur PTH/ PTHrP). Ces deux voies jouent un rôle majeur dans l'ossification enchondrale [3]. De plus, certaines des pathologies dues à des mutations de PTHRI fournissent un exemple de dysplasies liées à des troubles du métabolisme phosphocalcique. 


\section{Développement du squelette des vertébrés}

Le développement du squelette des vertébrés, soit plus de deux cent os formés de deux tissus (os et cartilage), met en jeu deux types d'ossification: enchondrale et membranaire. L'ossification enchondrale concerne les os longs, les vertèbres et quelques os de la base du crâne et dépend de l'existence d'une maquette cartilagineuse synthétisée par les chondrocytes. Elle repose sur une structure très particulière nécessaire à la croissance des os en longueur et à la différenciation de l'os primaire, la plaque de croissance. L'ossification membranaire ${ }^{1}$ concerne les os plats et se caractérise par une différenciation directe de cellules ostéoprogénitrices en pré-ostéoblastes puis en ostéoblastes matures dont certains deviennent des ostéocytes enclavés dans la matrice osseuse. Les deux types d'ossification font appel à des processus complexes incluant la migration et la condensation de cellules mésenchymateuses (morphogenèse) ainsi que leur différenciation en cellules cartilagineuses et/ou osseuses (organogenèse) [4]. Au niveau embryonnaire, les cellules mésenchymateuses chondrogéniques et ostéogéniques peuvent dériver de la crête neurale, des somites ou des plaques latérales du mésoderme [5]. Un troisième type cellulaire, l'ostéoclaste (ou chondroclaste), dérivé de la lignée hématopoïétique (monocyte/macrophage) est responsable de la résorption du tissu squelettique minéralisé. Les gènes impliqués dans la morphogenèse sont essentiellement des facteurs de transcription [6], tandis que l'organogenèse requiert des protéines de la matrice extracellulaire, des facteurs de signalisation et des facteurs transcriptionnels (Figure 1).

\section{Étapes et principaux facteurs impliqués dans le développement des membres}

\section{Formation du bourgeon de membre et facteurs de signalisation régulant sa croissance \\ Chez l'humain, la formation du bourgeon de mem- bre supérieur débute au $24^{e}$ jour de développe- ment embryonnaire et se manifeste sous la forme d'une protubérance issue du mésoderme latéral au niveau des sclérotomes C4 à C8. Le bourgeon de membre inférieur apparaît un peu plus tard à la fin de la $4^{\mathrm{e}}$ semaine de gestation. Chaque bourgeon se compose d'un noyau central de cel- lules mésenchymateuses d'origine mésodermique recouvertes d'une couche de cellules ectodermi- ques. L'extrémité du bourgeon montre la présence}

${ }^{1}$ Il est à noter que la clavicule s'ossifie en mettant en jeu les deux processus d'ossification, endochondrale et membranaire.

${ }^{2}$ Bien qu'il existe un FGF23 chez l'homme, le FGF15 n'a pas été identifié. La famille des FGF dans l'espèce humaine comporte ainsi 22 membres. d'un repli de cellules épithéliales formant la crête apicale ectodermique, située le long de l'axe antéro-postérieur (Figure 2). Cette structure est impliquée, via une interaction de type épithélio-mésenchymateuse, dans le maintien de la prolifération des cellules localisées sous la crête apicale qui restent indifférenciées et définissent la zone de progrès. Une troisième zone située postérieurement, appelée zone d'activité polarisante, détermine la polarité antéro-postérieure [7].

Parmi les facteurs de signalisation contrôlant la mise en place et la croissance du bourgeon de membre, le facteur le plus précocement exprimé est le FGF8, I'un des 22 membres de la famille des FGF (facteurs de croissance fibroblastique) identifiés chez l'homme? FGF8 est sécrété par les cellules de la crête apicale et semble responsable du maintien de la prolifération des cellules de la zone de progrès. De plus, il intervient dans l'induction de la zone polarisante en déclenchant l'expression de Sonic hedgehog (SHH), un médiateur de

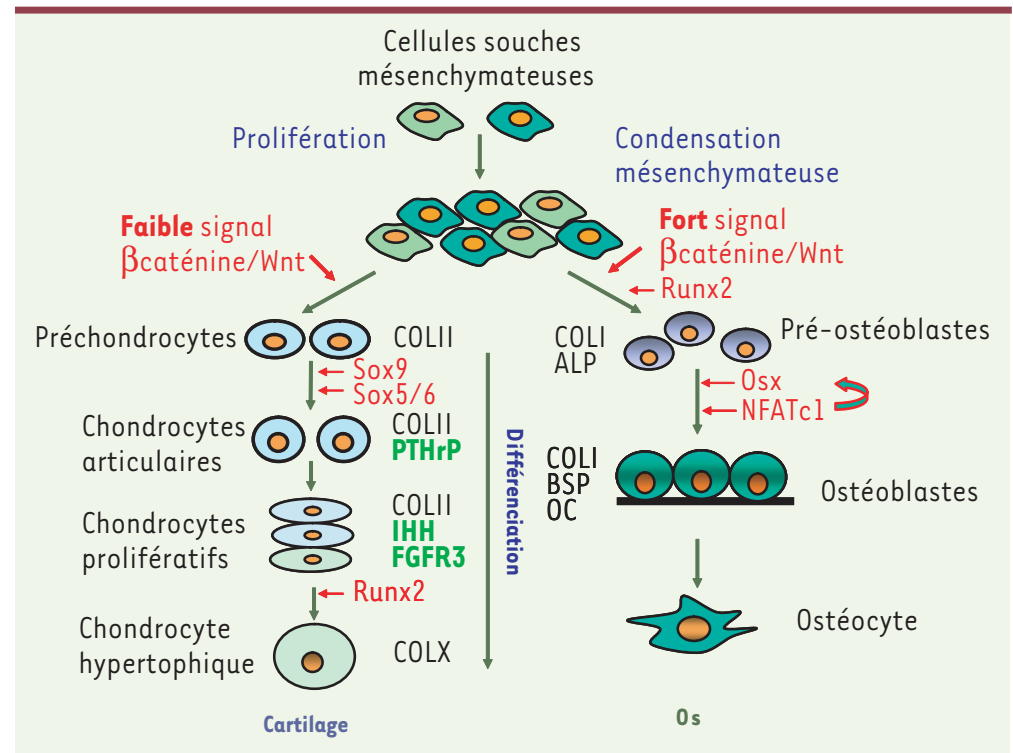

Figure 1. Formation et régulation par des facteurs transcriptionnels des lignées chondrocytaire et ostéoblastique. Après l'étape de condensation, l'intensité de la signalisation $\beta$-caténine/Wnt va déterminer la différenciation des cellules ostéoprogénitrices en chondrocytes ou en ostéoblastes. Les molécules de la matrice extracellulaire synthétisées par les différents types cellulaires sont notées en noir (COLI : collagène I; COL $I I$ : collagène II ; COL X : collagène X; BSP : bone sialoprotein ; OC : ostéocalcine; ALP : phosphatase alcaline) et les facteurs transcriptionnels régulant la différenciation des chondrocytes et des ostéoblastes sont notés en rouge (0sx: Osterix, facteur codé par le gène $S p 7$ et contenant un domaine en doigt de zinc ; NFATcl : nuclear factor of activated T cells) [6]. NFATcl régule l'activité transcriptionnelle de 0 sx. Les molécules de signalisation sont représentées en vert (PTHrP : parathyroid hormone related peptide; $\mathrm{IHH}$ : Indian hedgehog; FGFR3 : fibroblast growth factor receptor 3). Les ostéoblastes synthétisent une matrice osseuse dans laquelle certains ostéoblastes sont enclavés et convertis en ostéocytes. 


\begin{tabular}{|c|c|c|c|}
\hline Chondrodysplasie & Transmission & Gène & Type de protéine \\
\hline Achondrogenèse II (ACG2) & Dominante & COL2Al & Matrice extracellulaire \\
\hline Hypochondrogenèse (HCG) & Dominante & COL2Al & Matrice extracellulaire \\
\hline $\begin{array}{l}\text { Dysplasie spondyloépiphysaire congénitale } \\
\text { (SEDC) }\end{array}$ & Dominante & COL2AI & Matrice extracellulaire \\
\hline Syndrome de Kniest & Dominante & COL2AI & Matrice extracellulaire \\
\hline \multirow[t]{2}{*}{ Syndrome de Stickler } & Dominante & COL2Al & Matrice extracellulaire \\
\hline & Dominante & COLIIA2 & Matrice extracellulaire \\
\hline Syndrome de Marshall & Dominante & COLIIA2 & Matrice extracellulaire \\
\hline $\begin{array}{l}\text { Dysplasie oto-spondylo-méga épiphysaire } \\
\text { (OSMED) }\end{array}$ & Récessive & COLIIA2 & Matrice extracellulaire \\
\hline \multirow[t]{6}{*}{ Dysplasie poly-épiphysaire (MED) } & Dominante & COL9A1 & Matrice extracellulaire \\
\hline & Dominante & COL9A2 & Matrice extracellulaire \\
\hline & Dominante & COL9A3 & Matrice extracellulaire \\
\hline & Dominante & COMP & Matrice extracellulaire \\
\hline & Dominante & Matrilin 3 & Matrice extracellulaire \\
\hline & Récessive & DTDST & Transporteur de sulfate \\
\hline Dysplasie métaphysaire type Schmid (SCMD) & Dominante & COLIOAl & Matrice extracellulaire \\
\hline Pseudo-achondroplasie & Dominante & COMP & Matrice extracellulaire \\
\hline Achondrogenèse IB (ACGIB) & Récessive & DTDST & Transporteur de sulfate \\
\hline Atélostéogenèse 2 (A02) & Récessive & DTDST & Transporteur de sulfate \\
\hline Dysplasie diastrophique & Récessive & DTDST & Transporteur de sulfate \\
\hline Chondrodysplasie ponctuée & Liée à l’X & ARSE & Enzyme (arylsulfatase) \\
\hline Achondroplasie (ACH) & Dominante & FGFR3 & Récepteur tyrosine kinase \\
\hline Hypochondroplasie ( $\mathrm{HCH})$ & Dominante & FGFR3 & Récepteur tyrosine kinase \\
\hline Nanisme thanatophore (TD) & Dominante & FGFR3 & Récepteur tyrosine kinase \\
\hline Syndrome de Robinow & Récessive & ROR2 & Récepteur tyrosine kinase \\
\hline Brachydactylie type B & Dominante & ROR2 & Récepteur tyrosine kinase \\
\hline Dysplasie acro-mésomélique & Récessive & CDMPI & Facteur morphogénétique \\
\hline Brachydactylie type C & Dominante & CDMPI & Facteur morphogénétique \\
\hline Dysplasie acro-capito-fémorale & Récessive & $\mathrm{IHH}$ & Facteur de signalisation \\
\hline Brachydactylie type Al & Dominante & $\mathrm{IHH}$ & Facteur de signalisation \\
\hline $\begin{array}{l}\text { Dysplasie métaphysaire } \\
\text { type Jansen (JMC) }\end{array}$ & Dominante & PTHRI & Récepteur hormonal \\
\hline Dysplasie de Blomstrand & Récessive & PTHRI & Récepteur hormonal \\
\hline Dysplasie campomélique & Dominante & sox 9 & Facteur transcriptionnel \\
\hline Dyschondrostéose & Pseudo-autosomique & SHOX & Facteur transcriptionnel \\
\hline Dysplasie chondro- ectodermique ( $\varepsilon v c)$ & Récessive & $\varepsilon V C 1$ & Facteur transcriptionnel \\
\hline & & $\varepsilon V C 2$ & Facteur transcriptionnel \\
\hline
\end{tabular}

Tableau I. Liste des chondrodysplasies dont le gène responsable et la protéine correspondante ont pu être identifiés et partiellement caractérisés. 
la polarisation antéro-postérieure [8]. FGF8 est également capable d'induire l'expression de FGF10 qui s'exprime dans la partie distale du mésenchyme du bourgeon et assure une régulation en retour de l'expression de FGF8. Cette régulation réciproque des deux facteurs s'effectue par le biais de FGFR2, un des récepteurs des FGF pour lequel deux isoformes majeurs ont été identifiées. L'isoforme IIlb s'exprime dans les cellules épithéliales et se lie au FGF10, alors que l'isoforme IIlc s'exprime dans les cellules mésenchymateuses et se lie au FGF8 [9]. D'autres facteurs sont synthétisés par les cellules ectodermiques dorsales notamment engrailed 1 ( $(\varepsilon n-1)$ et Wnt7a. Ce dernier agit par l'intermédiaire de Lmx-l exprimé dans le mésoderme sous-jacent pour contrôler la formation des membres selon l'axe dorso-ventral [7]. L'ensemble de ces facteurs définit un réseau de signalisation impliqué dans la mise en place des trois axes de symétrie du membre (Figure 2). Ces mêmes facteurs sont capables de réprimer leur propre activité en induisant la synthèse d'antagonistes ( $\mathrm{Pbx}$, Meis) formant des complexes avec les gènes Hox exprimés dans la partie distale du membre, notamment les gènes Hoxal3 et Hoxdl3, pour assurer un contrôle strict de la croissance des membres [10].

\section{Condensation des cellules mésenchymateuses}

L'étape de condensation des cellules mésenchymateuses pour former les éléments préchondrogéniques joue un rôle décisif dans l'architecture finale de chacun des éléments du squelette, mais reste mal comprise au niveau moléculaire. L'initiation de la condensation dans la région proximale du membre supérieur va permettre la formation de l'humérus, puis le processus se propage selon l'axe proximo-distal pour générer le radius et le cubitus. Finalement, par des mécanismes de branchement et de segmentation, les carpes, métacarpes et phalanges apparaissent. Les mêmes étapes, décalées dans le temps, opèrent pour le membre inférieur. La condensation des cellules souches mésenchymateuses repose sur des interactions cellules-cellules qui établissent un centre d'agrégation capable de recruter des cellules à partir des tissus environnants. Ce processus implique plusieurs classes de molécules dont la N-cadhérine et la N-CAM [11]. Le processus de différenciation de ces cellules ostéoprogénitrices en chondrocytes ou en ostéoblastes dépend de la voie Wnt/ $\beta$ caténine [12] (Figure 1). Le facteur transcriptionnel Sox-9 joue également un rôle majeur dans la différenciation chondrocytaire puisque son inactivation dans le bourgeon de membre de la souris empêche la condensation et conduit à une mort cellulaire accélérée des cellules mésenchymateuses. Son expression serait contrôlée par les FGF et il est lui-même un régulateur de l'expression des gènes codant pour les collagènes de type II et XI ainsi que des gènes Sox-5 et Sox- 6 impliqués dans la formation du cartilage $[13,14]$. Ces fonctions multiples expliquent en partie pourquoi des mutations perte de fonction de Sox-9 sont responsables chez l'homme d'une chondrodysplasie létale, la dysplasie campomélique [15].

\section{Plaque de croissance et chondrogenèse}

Au fur et à mesure que progresse la condensation des cellules mésenchymateuses en chondrocytes, il devient possible d'identifier certaines zones correspondant à des sous-populations de chondrocytes à différents stades de différenciation (Figure 3). Les chondrocytes localisés à l'extrémité de l'épiphyse sont immatures et définissent la zone de réserve. Les cellules les plus distales ont la capacité d'entrer en division pour engendrer la zone proliférative qui montre un empilement de cellules formant des groupes isogéniques. Les chondrocytes de la partie distale de cette zone cessent de proliférer et commencent à se différencier en chondrocytes préhypertrophiques dont la taille est supérieure à celle des chondrocytes prolifératifs. La différenciation se poursuit et s'accompagne d'un accroissement de la taille des cellules qui deviennent hypertrophiques. La zone d'hypertrophie, dont la taille varie au cours du développement fœtal,

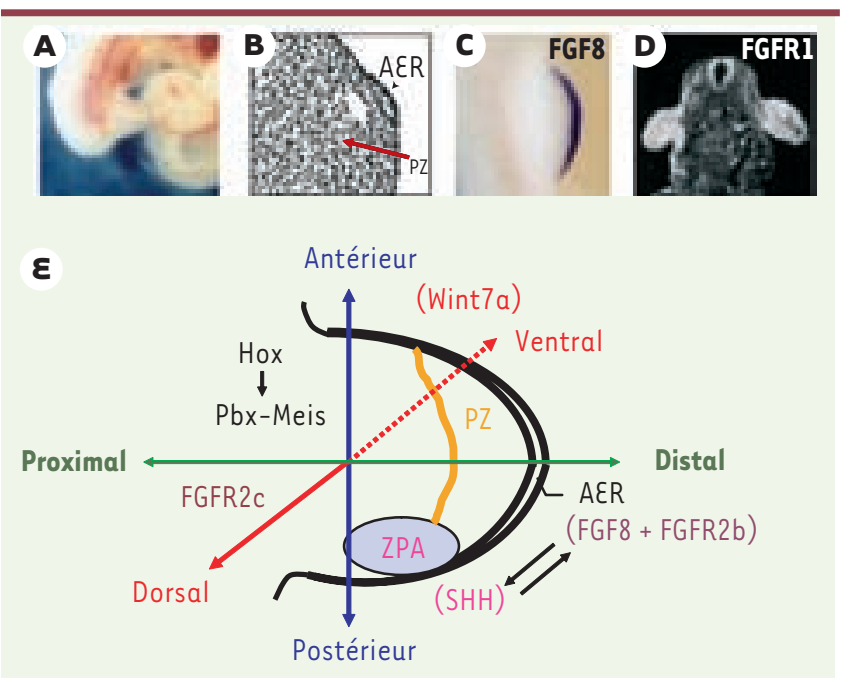

Figure 2. Organisation du bourgeon de membre et voies de signalisation impliquées dans sa mise en place. A. Bourgeons de membres inférieurs chez un embryon humain de 30 jours. $\boldsymbol{B}$. Coupe transversale du bourgeon de membre montrant la crête apicale ectodermique (AER) et la zone de progrès (PZ). C. Expression de FGF8 au niveau de la crête apicale ectodermique. D. Expression de FGFRI (fibroblast growth factor receptor 1 ) dans les cellules mésenchymateuses des bourgeons de membres supérieurs (embryon de 30 jours). $\varepsilon$. Développement spatial du bourgeon selon les trois axes: antéro-postérieur, dorso-ventral et proximo-distal. Les cellules de la crête apicale ectodermique (AER) sécrètent le FGF8 qui active SHH (Sonic hedgehog) au niveau de la zone d'activité polarisante (ZPA). SHH exerce un rétro-contrôle sur l'expression des FGF (facteurs de croissance fibroblastique). Wnt7a est exprimé au niveau de l'ectoderme dorsal et régule l'expression de Lmx-ldans le mésenchyme dorsal. Les homéoprotéines Pbx et Meis (myeloid ecotropic insertion site) forment des complexes capables d'interagir avec les produits de certains gènes Hox pour les réguler négativement et favoriser l'activation de la boucle FGF/SHH. 
serait, selon certains auteurs, le moteur essentiel de la croissance osseuse [3]. Les chondrocytes hypertrophiques en différenciation terminale vont contrôler la minéralisation de leur matrice et entrer en apoptose. Les travées calcifiées laissées libres sont progressivement envahies par les ostéoblastes et les canaux vasculaires pour former une véritable matrice d'os spongieux (spongiosa). À chaque stade, les cellules expriment des marqueurs spécifiques. Les chondrocytes de la zone de repos et de la zone proliférative synthétisent majoritairement le collagène de type II et l'agrécane et en plus faible quantité les collagènes de type IX et $\mathrm{XI}$ ainsi que des protéines non collagéniques (COMP ou cartilage oligomeric matrix protein, matrilin, chondroadherin, fibromodulin...), tandis que les chondrocytes hypertrophiques synthétisent du collagène de type $X$. La progression phénotypique linéaire des chondrocytes au niveau de cette structure hautement différenciée qu'est la plaque de croissance est un processus finement régulé. Deux voies de signalisation jouent un rôle essentiel dans la coordination de la prolifération et de la différenciation des chondrocytes, la voie régie par le FGFR3 et celle régie par le PTHRI [16-18]. Ces conclusions reposent sur les études de chondrodysplasies dues à des mutations des gènes FGFR3 et PTHRI et l'analyse de souris génétiquement modifiées.

\section{Chondrodysplasies et voies de signalisation transmises par le FGFR3 et le PTHRI}

\section{Récepteur FGFR3 et ossification enchondrale}

FGFR3 fait partie d'une famille de quatre récepteurs à activité tyrosine kinase qui présentent une organisation structurale commune incluant un domaine extracellulaire avec trois boucles de type immunoglobuline (Ig), un domaine transmembranaire hydrophobe d'ancrage du récepteur dans la membrane et un domaine intracellulaire responsable de l'activité kinase. Ces récepteurs fixent normalement les FGF au niveau de la troisième boucle Ig. Les études tridimensionnelles ont montré que deux molécules de ligand sont nécessaires pour induire la dimérisation du récepteur, l'ensemble formant un complexe ternaire dans lequel un protéoglycane de surface à base de sulfate d'héparane (HSPG) agit en qualité de corécepteur [19]. FGFR3 est exprimé au niveau de la plaque de croissance par les chondrocytes de la zone proliférative et de préhypertrophie.

\section{Chondrodysplasies dues}

\section{à des mutations du gène FGFR3}

La démonstration qu'une mutation ponctuelle récurrente du gène FGFR3 était responsable de la quasi- totalité des cas d'achondroplasie [20] a ouvert un nouveau champ d'investigation en pathologie osseuse. À l'instar de l'achondroplasie, des mutations ponctuelles récurrentes affectant différents domaines de FGFR3 ont été identifiées chez des patients atteints d'hypochondroplasie, de syndrome SADDAN ou de nanisme thanatophore de type I et II [21, 22]. Histologiquement, I'ampleur des anomalies de la plaque de croissance chez les patients reflète la sévérité de la maladie (Figure 3 ). La réduction de la zone proliférative s'accompagne d'une perte de l'organisation des chondrocytes en colonne et de la présence de chondrocytes de petite taille dans la zone d'hypertrophie suggérant une anomalie de la différenciation [23]. Les mutations de FGFR3 localisées dans le domaine extracellulaire créent des résidus cystéines surnuméraires capables de former des ponts disulfures entre deux molécules de récepteur mutant. Les mutations du domaine catalytique entraînent une modification de la conformation du domaine qui semble interférer avec le transport vers la membrane plasmique du récepteur muté [24]. Dans les deux cas, il en résulte une phosphorylation constitutive du récepteur en l'absence de ligand et une activation de la voie de signalisation STAT

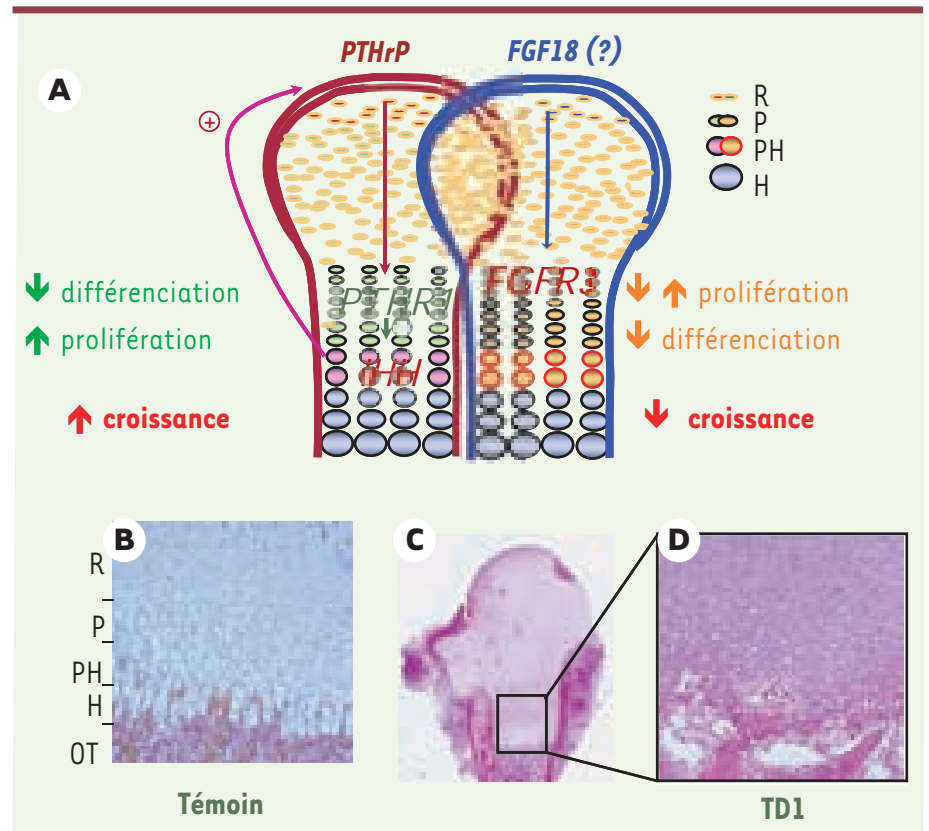

Figure 3. Schéma de la plaque de croissance d'un os long. A. Plaque de croissance. Deux voies principales de signalisation sont impliquées dans la régulation de la prolifération et de la différenciation des chondrocytes, la voie IHH/PTHRI et la voie FGF/FGFR, détaillées dans le corps de l'article. PTHrP: parathyroid hormone related peptide; PTHRI : parathyroid hormone receptor 1 ; $\mathrm{IHH}$ : Indian hedgehog. B. Coupe de cartilage fémoral d'un fœtus témoin de 25 semaines montrant les différentes zones de la plaque de croissance. $R$ : zone de repos ; $\mathrm{P}$ : zone proliférative ; $\mathrm{PH}$ : zone de pré-hypertrophie ; $\mathrm{H}$ : zone d'hypertrophie; OT: os trabéculaire. C. Coupe de cartilage d'un fœtus de 24 semaines atteint de nanisme thanatophore de type I (TD I) porteur d'une mutation de FGFR3 (S249C). D. Grossissement illustrant la désorganisation de la plaque de croissance. 
(signal transducers and activators of transcription). Les molécules Stat 1,3 et 5 sont phosphorylées et transloquées dans le noyau où elles activent l'inhibiteur des cyclines kinases-dépendantes p21 $1^{\mathrm{CIPl}}$. Cette activation ferait entrer de façon prématurée les cellules de la zone proliférative dans la voie d'hypertrophie. Cependant, ces chondrocytes préhypertrophiques semblent incapables d'atteindre la phase ultime de différenciation impliquée dans l'élongation de l'os. Une mort prématurée par apoptose pourrait expliquer leur incapacité à s'hypertrophier efficacement. Ces conclusions tirées des travaux sur le cartilage et les chondrocytes de patients atteints de nanisme thanatophore sont confortées par les résultats de l'étude de modèles murins dans lesquels les mutations responsables de l'achondroplasie et du nanisme thanatophore ont été reproduites [25-27]. L'ensemble de ces résultats, ainsi que le phénotype des souris invalidées pour le gène Fgfr3, confirment le rôle de régulateur négatif de la croissance osseuse de FGFR3 [22].

\section{Voie de signalisation PTHR1}

Le PTHRI appartient à la famille II de récepteurs à sept domaines transmembranaires couplés aux protéines $G$ hétérotrimériques. Ce récepteur assure les fonctions endocrines de la PTH et celles autocrines/paracrines du PTHrP. La PTH joue un rôle essentiel dans le maintien de l'homéostasie phosphocalcique. Le PTHrP, décrit initialement dans des hypercalcémies humorales malignes, n'est pas détectable physiologiquement dans la circulation systémique. Comme le PTHRl, il est exprimé dans de nombreux tissus et exerce de multiples actions physiologiques qu'il n'est pas possible de résumer dans cette revue (voir [28] et revue de J.J. Helwig à paraître dans médecine/sciences). En particulier, c'est un régulateur de la prolifération et de la différenciation cellulaire, notamment lors du développement osseux enchondral. Ses effets biologiques sur la prolifération cellulaire dans certains types de cellules sont opposés, suivant qu'il agit de façon auto/paracrine via la stimulation du PTHRl ou intracrine via sa translocation dans le noyau des cellules. Dans le tissu squelettique, il est établi que l'activation du PTHRl (exprimé par les chondrocytes prolifératifs et préhypertrophiques) par le PTHrP sécrété par les chondrocytes périarticulaires et les cellules périchondriales stimule la prolifération des chondrocytes préhypertrophiques et inhibe leur différenciation. La production du PTHrP est stimulée par Indian hedgehog $(\mathrm{IHH})$, un morphogène membre de la famille hedgehog, qui est fortement exprimé dans la zone de transition entre chondrocytes préhypertrophiques et hypertrophiques. L'activation du PTHRl entraîne donc une diminution de différenciation des cellules productrices de $\mathrm{IHH}$, assurant ainsi un contrôle très fin de l'équilibre entre prolifération et différenciation au niveau de la plaque de croissance. Certains modèles murins suggèrent l'existence d'une interaction entre les voies PTHRI/FGFR3 qui ne serait pas présente chez l'homme [29].

\section{Chondrodysplasies associées à des mutations du PTHR1}

Quatre chondrodysplasies rares, mais dont le phénotype illustre les rôles endocrines et paracrines assurés par le PTHRl, ont été associées à des mutations de ce gène.
Les mutations activatrices du PTHRI causent la chondrodysplasie métaphysaire de Jansen [30]. Cette chondrodysplasie, de transmission autosomique dominante, est caractérisée par un nanisme sévère et des anomalies biologiques qui sont celles rencontrées chez les patients atteints d'hyperparathyroïdie primaire ou du syndrome d’hypercalcémie humorale maligne. Les lésions métaphysaires observées radiologiquement rappellent celles du rachitisme par carence en vitamine $D$ et témoignent d'une augmentation de prolifération et d'un retard de différenciation des chondrocytes (élargissement des métaphyses, présence de structures irrégulières inégalement calcifiées). Quatre mutations hétérozygotes faux-sens ont été identifiées chez des patients atteints de chondrodysplasie de Jansen. Une corrélation génotype/phénotype est décrite en fonction du degré d'activation constitutive démontrée in vitro. En accord avec les anomalies métaphysaires des patients atteints de chondrodysplasie de Jansen, les souris transgéniques exprimant dans les chondrocytes prolifératifs le PTHRI humain porteur de la mutation His223Arg, la plus fréquente et la plus sévère, montrent un retard majeur de différenciation des chondrocytes au niveau de la plaque de croissance.

Les mutations inactivatrices du PTHRl sont responsables de la chondrodysplasie de Blomstrand [30]. Image en miroir de la chondrodysplasie de Jansen, cette dysplasie, de transmission récessive, est létale et caractérisée par un syndrome dysmorphique marqué par une extrême brièveté des membres, une avance majeure de l'ossification enchondrale avec hyperdensité osseuse. Ce phénotype est semblable à celui observé chez les souris invalidées de façon homozygote pour les gènes PTHrP ou PTHR1. Quatre mutations perte de fonction (faux sens, anomalie d'épissage, décalage du cadre de lecture, délétion de la région promotrice) ont été rapportées. Comme pour les mutations activatrices, une corrélation génotype/phénotype a été décrite en fonction du degré d'inactivation du récepteur in vitro, le phénotype étant toujours létal [30].

Très récemment, une mutation du gène PTHRl, que nous appellerons «régulatrice», a été identifiée dans une forme familiale récessive de chondrodysplasie de Eiken. Cette pathologie se caractérise par une dysplasie épiphysaire multiple et un retard majeur de l'ossification endochondrale avec anomalies du modelage et persistance de cartilage dans certains os, sans anomalie du métabolisme phosphocalcique. On a donc une transmission récessive avec des signes cliniques compatibles avec une activation constitutive du PTHRl, mais sans hyperparathyroïdie. La mutation identifiée, Arg485X, entraîne la 
délétion des 108 derniers acides aminés du PTHRl. Le récepteur tronqué serait responsable d'un déséquilibre entre les deux voies de transduction ou d'une anomalie de trafficking [31].

La dernière pathologie associée à une mutation du gène PTHRI est la maladie d'Ollier. La maladie d'Ollier, ou enchondromatose, est définie par la présence de tumeurs cartilagineuses bénignes qui se développent dans l'os. II s'agit d'une affection le plus souvent sporadique, cependant des formes familiales ont été rapportées. Les résultats de l'analyse moléculaire suggèrent une hétérogénéité génétique de l'enchondromatose. Une mutation (Arg150Cys) dans le gène PTHRI a été identifiée chez deux malades atteints de maladie d'Ollier [32]. Le récepteur mutant PTHRI Arg150Cys paraît être activé de façon constitutive, cependant la mutation est associée à un défaut d'expression à la membrane cellulaire. Les souris transgéniques exprimant de façon ciblée le récepteur PTHRl mutant dans les chondrocytes pré-hypertrophiques développent des tumeurs similaires à celles observées chez les patients atteints d'enchondromatose, tout comme les souris surexprimant le régulateur transcriptionnel Gli2, régulateur en aval de IHH. II apparaît donc que l'activation de la voie IHH, PTHrP, PTHRl joue un rôle important dans le développement des enchondromes. II faut souligner que les malades atteints d'une chondrodysplasie de Jansen, donc associée à une mutation activatrice du récepteur PTHRl, ne présentent pas d'enchondromes. À l'inverse, contrairement aux malades atteints de chondrodysplasie de Jansen, les malades atteints d'enchondromatose associée à une mutation du gène PTHRI (même germinale) ne présentent pas d'anomalies du métabolisme phosphocalcique. L'origine de ces différences n'est pas connue.

\section{Conclusions}

L'étude de dysplasies squelettiques rares affectant le développement et la croissance osseuse a permis de localiser et d'identifier certains des gènes responsables (plus de 75 à ce jour) et de révéler de nouvelles voies de signalisation impliquées dans les processus d'ossification enchondrale et membranaire. Outre les voies contrôlées par les récepteurs FGFR3 et PTHRl, des travaux récents ont démontré le rôle clé de la voie $\beta$-caténine/Wnt dans la différenciation chondrocytaire et ostéoblastique. Néanmoins, face à un nombre croissant de nouveaux gènes dont les fonctions régulatrices dans la formation osseuse sont encore mal comprises, il est nécessaire d'établir les relations précises entre les produits de ces gènes et ceux déjà connus et de déterminer comment les différentes voies de signalisation interagissent entre elles et sont affectées par des mutations de ces gènes. Les modèles murins dans lesquels il est possible, grâce au système Cre- $\varepsilon R$, d'induire de façon tissu spécifique et à un moment précis du développement des mutations identiques à celles identifiées chez l'homme, offrent de nouvelles perspectives pour mieux comprendre les phénotypes observés. Cette connaissance précise des mécanismes intimes de la croissance osseuse est un préalable indispensable à la mise au point de thérapies médicamenteuses ou cellulaires destinées à corriger les dysplasies du squelette. $\diamond$

\section{SUMMARY}

Hereditary skeletal dysplasias and FGFR3 and PTHRI signaling pathways

Skeletal development is a highly sophisticated process involving, as a first step, migration and condensation of mesenchymal cells into osteoprogenitor cells. These cells further differentiate into chondrocytes and osteoblasts through multiple differentiation stages requiring a set of specific transcriptional factors. Defective endochondral ossification in human is associated with a large number of inherited skeletal dysplasias caused by mutations in genes encoding extracellular matrix components, growth factors and their receptors, signaling molecules and transcription factors. This review summarizes some of the recent findings on a series of chondrodysplasias caused by mutations in FGFR3 and PTHRl, two receptors expressed in the cartilage growth plate and mediating two main signaling pathways. Data from human diseases and relevant animal models provide new clues for understanding how signaling molecules and their interaction with key transcription factors control and regulate the development and growth of long bones. $\diamond$

\section{RÉFÉRENCES}

1. Hall CM. International nosology and classification of constitutional disorders of bone (2001). Am J Med Genet $2002 ; 113: 65-77$.

2. Chapman KL, Mortier GR, Chapman K, et al. Mutations in the region encoding the von Willebrand factor A domain of matrilin-3 are associated with multiple epiphyseal dysplasia. Nat Genet $2001 ; 28: 393-6$.

3. Kronenberg HM. Developmental regulation of the growth plate. Nature $2003 ; 423: 332-6$.

4. Karsenty $G$. The complexities of skeletal biology. Nature $2003 ; 423: 316-8$.

5. Zelzer $\varepsilon$, Olsen BR. The genetic basis for skeletal diseases. Nature $2003 ; 423: 343-8$.

6. Kobayashi T, Kronenberg H. Minireview : transcriptional regulation in development of bone. Endocrinology $2005 ; 146: 1012-7$.

7. Johnson RL, Tabin CJ. Molecular models for vertebrate limb development. Cell 1997 ; 90 : 979 90.

8. Lewandoski M, Sun X, Martin GR. Fgf8 signalling from the AER is essential for normal limb development. Nat Genet $2000 ; 26: 460-3$.

9. Xu X, Weinstein M, Li C, et al. Fibroblast growth factor receptor 2 (FGFR2)-mediated reciprocal regulation loop between FGF8 and FGF10 is essential for limb induction. Development 1998 ; $125: 753-65$

10. Vogt TF, Duboule D. Antagonists go out on a limb. Cell $1999 ; 99: 563-6$.

11. Hall BK, Miyake T. All for one and one for all : condensations and the initiation of skeletal development. Bioessays $2000 ; 22$ : 138-47.

12. Kolpakova $\varepsilon$, Olsen BR. Wnt/beta-catenin: a canonical tale of cell-fate choice in the vertebrate skeleton. Dev Cell $2005 ; 8: 626-7$.

13. Akiyama H, Chaboissier MC, Martin JF, et al. The transcription factor Sox 9 has essential roles in successive steps of the chondrocyte differentiation pathway and is required for expression of Sox 5 and Sox6. Genes Dev $2002 ; 16: 2813-28$.

14. Bi W, Deng JM, Zhang Z, et al. Sox9 is required for cartilage formation. Nat Genet 1999 ; 22 : 85-9. 
15. Wagner T, Wirth J, Meyer J, et al. Autosomal sex reversal and campomelic dysplasia are caused by mutations in and around the SRY-related gene SOX9. Cell $1994 ; 79: 1111-20$.

16. Long F, Zhang XM, Karp S, et al. Genetic manipulation of hedgehog signaling in the endochondral skeleton reveals a direct role in the regulation of chondrocyte proliferation. Development 2001 ; 128 : 5099108.

17. Karsenty $G$, Wagner $E F$. Reaching a genetic and molecular understanding of skeletal development. Dev Cell $2002 ; 2: 389-406$.

18. Beier F, Ali Z, Mok D, et al. TGFbeta and PTHrP control chondrocyte proliferation by activating cyclin Dl expression. Mol Biol Cell 2001; 12 : 3852-63.

19. Schlessinger J. Cell signaling by receptor tyrosine kinases. Cell 2000 ; $103: 211-25$

20. Rousseau F, Bonaventure J, Legeai-Mallet L, et al. Mutations in the gene encoding fibroblast growth factor receptor-3 in achondroplasia. Nature $1994 ; 371: 252-4$.

21. Vajo Z, Francomano CA, Wilkin DJ. The molecular and genetic basis of fibroblast growth factor receptor 3 disorders : the achondroplasia family of skeletal dysplasias, Muenke craniosynostosis, and Crouzon syndrome with acanthosis nigricans. Endocrinol Rev $2000 ; 21: 23-39$.

22. Ornitz DM, Marie PJ. FGF signaling pathways in endochondral and intramembranous bone development and human genetic disease. Genes Dev $2002 ; 16$ : 1446-65.

23. Legeai-Mallet L, Benoist-Lasselin C, Munnich A, et al. Overexpression of FGFR3, Statl, Stat5 and p21Cipl correlates with phenotypic severity and defective chondrocyte differentiation in FGFR3-related chondrodysplasias. Bone $2004 ; 34: 26-36$.

24. Lievens PM, Mutinelli C, Baynes D, et al. The kinase activity of fibroblast growth factor receptor 3 with activation loop mutations affects receptor trafficking and signaling. J Biol Chem $2004 ; 279$ : 43254-60.

25. Segev 0, Chumakov I, Nevo Z, et al. Restrained chondrocyte proliferation and maturation with abnormal growth plate vascularization and ossification in human FGFR-3(G380R) transgenic mice. Hum Mol Genet $2000 ; 9: 249-58$.

26. Iwata T, Chen L, Li C, et al. A neonatal lethal mutation in FGFR3 uncouples proliferation and differentiation of growth plate chondrocytes in embryos. Hum Mol Genet 2000 ; 9 : 1603-13.

27. Chen L, Li C, piao W, et al. A Ser(365)->Cys mutation of fibroblast growth factor receptor 3 in mouse downregulates Ihh/PTHrP signals and causes severe achondroplasia. Hum Mol Genet $2001 ; 10:$ 457-65.

28. Clemens TL, Cormier S, Eichinger A, et al. Parathyroid hormone-related protein and its receptors: nuclear functions and roles in the renal and cardiovascular systems, the placental trophoblasts and the pancreatic islets. BrJ Pharmacol $2001 ; 134: 1113-36$.

29. Cormier S, Delezoide AL, Benoist-Lasselin C, et al. Parathyroid hormone receptor type $1 /$ Indian hedgehog expression is preserved in the growth plate of human fetuses affected with fibroblast growth factor receptor type 3 activating mutations. Am J Pathol 2002; 161 : 1325-35.

30. Jüppner $H$, Schipani $\varepsilon$, Silve $C$. Jansen's metaphyseal chondrodysplasia and Blomstrand's lethal chondrodysplasia : two genetic disorders caused by PTH/PTHrP receptor mutations. In : Bilezikian J, Raisz L, Rodan G, eds. Principles of bone biology, vol. 2. San Diego, CA : Academic Press, 2002 : 1117-35.

31. Duchatelet $S$, Ostergaard $\varepsilon$, Cortes $D$, et al. Recessive mutations in PTHRI cause contrasting skeletal dysplasias in Eiken and Blomstrand syndromes. Hum Mol Genet 2005 ; 14 : 1-5.

32. Hopyan S, Gokgoz N, Poon R, et al. A mutant PTH/PTHrP type I receptor in enchondromatosis. Nat Genet $2002 ; 30$ : 306-10.

\section{TIRÉS À PART}

J. Bonaventure

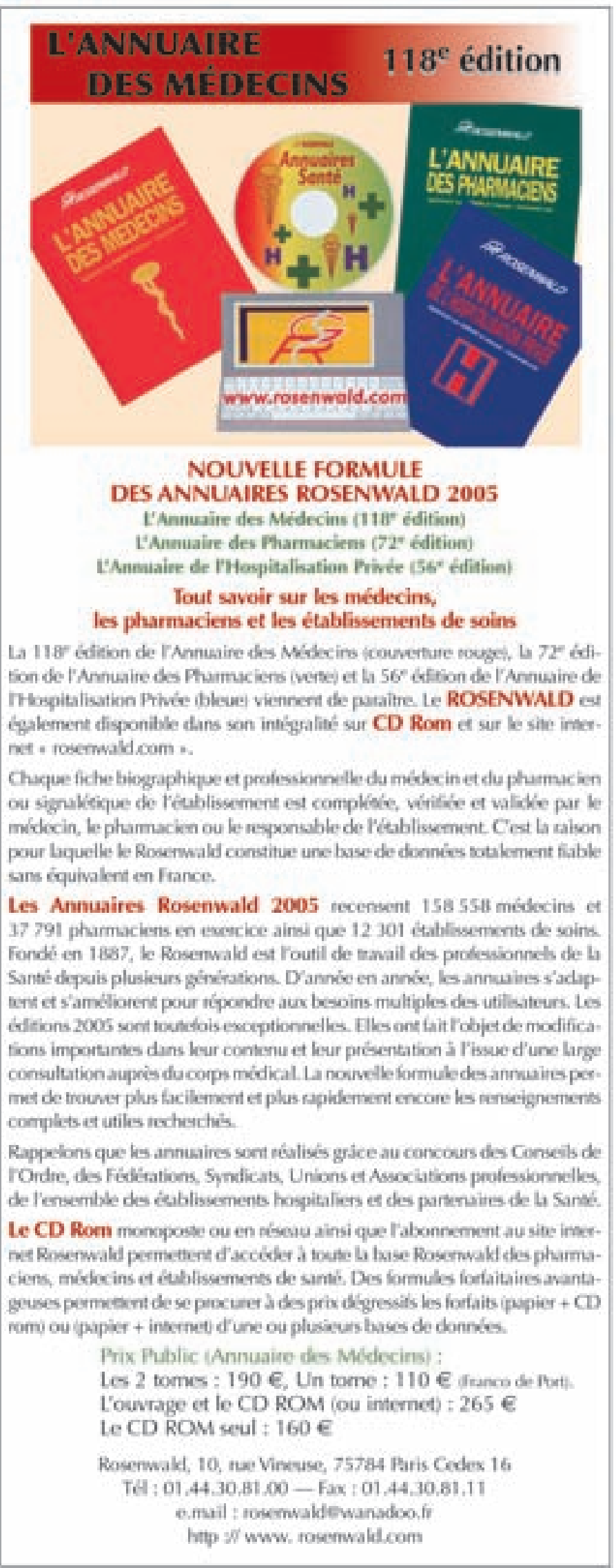

